\title{
Level Set Based Integration of Segmentation and Computational Fluid Dynamics for Flow Correction in Phase Contrast Angiography
}

\author{
Masao Watanabe ${ }^{1,2}$, Ron Kikinis ${ }^{1}$, and Carl-Fredrik Westin ${ }^{1}$ \\ 1 Laboratory of Mathematics in Imaging, Brigham and Women's Hospital \\ Harvard Medical School, Boston MA, USA \\ \{watanabe, kikinis, westin\}@bwh.harvard.edu \\ 2 Dept. of Mechanical Engineering, Kyushu University, Fukuoka, Japan \\ watanabe@mech.kyushu-u.ac.jp
}

\begin{abstract}
A novel approach to correct flow data from phase contrast angiography (PCA) is presented. The method is based on combining computational fluid dynamics (CFD) and segmentation in a level set framework. The PCA-MRI velocity data is used in a partial differential equation (PDE) based level set method for vessel segmentation, and a second level set equation solving for a physically meaningful flow. The second level set is implemented using the ghost fluid method, where the MR data defines initial and boundary conditions. The segmentation and CFD systems are simultaneously integrated to provide a robust method yielding a physically correct velocity and optimal vessel geometry. The application of this system to both synthetic and clinical data is demonstrated and its validity is discussed.
\end{abstract}

\section{Introduction}

In 3D phase contrast angiography (PCA) sequences, the velocities of blood flow in three orthogonal directions are mapped to phase differences, which is controlled by a variable known as "velocity encoding" or venc [1. This sequence results in phase wrapping in areas of flow with greater speed than the venc. As an additional complication, signal quality typically deteriorates because of phase dispersion from turbulence and vortices stemming from pulse or vessel branching. These artifacts impede accurate flow quantification, especially with respect to flow direction. The need to establish vessel diameter is also critical; but because PCA-MRI sequences produce very weak MR signals in the neighborhood of vessel wall, signal resolution in this region predictably degenerates.

Computational approaches based on MR segmentation have previously been applied in arterial biomechanics [2, hemodynamics of carotid artery bifurcations [3], and general vascular segmentation using level set methods [4]. Combined computational fluid dynamics (CFD) and MRI studies have been conducted on the reconstruction of blood flow patterns in a human carotid bifurcation [5]. These studies generally employ complicated, unstructured CFD grid systems 
constructed from medical images [6], and use the MRI data only for the segmentation, i.e., grid system generation. The available velocity information in PCA-MRI is in general not applied in CFD studies of blood flow.

We have developed a numerical scheme to model blood flow in vessels by solving the incompressible Navier-Stokes equation with vessel geometry segmented by a partial differential equation (PDE) based fast local level set method [7. By implementing the level set Ghost Fluid Method (GFM) [8], we have effectively enforced a zero-velocity boundary condition on the vessel wall (the zero level set) without smearing physical properties near the wall. This approach has enabled us to use a simple structured computational grid. The improvement of velocity fields is verified for both synthetic and clinical data.

\section{Numerical Formulation}

For the purpose of characterizing blood flow in vessels, we have developed a numerical scheme to model incompressible fluid flow in a tubular flow path, bounded by a solid, rigid, wall. This approach enables us to define the solid tubular wall boundary as the interface between the incompressible fluid and the rigid solid, and to solve a stationary interface problem by applying the proven level set method. The level set method was originally developed by Osher and Sethian [9] as a simple and versatile method for computing and analyzing the motion of an interface in two or three dimensions, such as, for example, computing two-phase Navier-Stokes incompressible flows [10]. However, the original level set method smears out both the density and the viscosity across the interface, in order to prevent spurious oscillatory solutions at the interface. As explained in [11], the original GFM was developed to solve this problem by populating cells next to the interface with "ghost values", and extrapolating values across the interface.

In this work, we couple the incompressible Navier-Stokes equation solver with high accuracy, combining the level set scheme with a projection method developed by Sussman et al. [12, with the GFM developed by Fedkiw et al. [8]. This has resulted in a stable zero-velocity boundary condition on the vessel wall.

\subsection{Governing Equations}

At each time step, we solve the following dimensionless evolution equations for the velocity and pressure,

$$
\begin{gathered}
\boldsymbol{\nabla} \cdot \boldsymbol{u}=\mathbf{0}, \\
\frac{\partial \boldsymbol{u}}{\partial t}+\boldsymbol{u} \cdot(\boldsymbol{\nabla} \boldsymbol{u})=-\boldsymbol{\nabla} p+\frac{1}{\operatorname{Re}} \nabla^{2} \boldsymbol{u},
\end{gathered}
$$

where $t$ is time, $\boldsymbol{u}$ is velocity, $p$ is pressure. The dimensionless parameter, Re, used in Eq. (2) is the Reynolds number $(\operatorname{Re}=\rho L U / \mu)$, where $L$ and $U$ are the characteristic length and velocity, respectively, $\rho$ and $\mu$ are density and viscosity of blood, respectively. We used the values $1.055 \times 10^{3} \mathrm{~kg} / \mathrm{m}^{3}$ for $\rho$, and $4.50 \times$ $10^{-3} \mathrm{~kg} / \mathrm{m} \mathrm{s}$ for $\mu$. 


\subsection{Discretization and Time Integration}

We follow the discretization methodology and time integration procedure that Sussman et al. developed [12]. This scheme implements essentially a non-oscillatory third order method to evaluate the convection term and the fractional time step projection method, while enforcing that the continuity equation (1D) is satisfied. These methods guarantee stability in high velocity fields, robustness in complicated geometries, and give high accuracy without smearing the solution.

\subsection{Solid Wall Conditions Using Ghost Cells}

The ghost cells are defined in the solid-side neighborhood of the fluid-solid interface (i.e. vessel wall) 8]. We can modify pressure in the ghost cells by using the isobaric fix technique [8], by defining the unit normal at every grid point as $\boldsymbol{N}=\nabla \phi /|\nabla \phi|$ and then solving a partial differential equation for "constant extrapolation" in the normal direction. The equation is

$$
\frac{\partial p}{\partial \tau}+\boldsymbol{N} \cdot \boldsymbol{\nabla} p=0 .
$$

We have developed the zero-velocity fix on the solid wall, by simple extension of the isobaric fix technique. We consider the new variable $\boldsymbol{v} ; \boldsymbol{v}=\boldsymbol{u} / \phi$. First, the constant extrapolation of $\boldsymbol{v}$ is calculated in the direction of the normal to the solid wall in the neighborhood of the wall. Then the zero velocity fix is completed by setting $\boldsymbol{u}=\boldsymbol{v} \phi$. The partial differential equation governing $\boldsymbol{v}$ to be solved is the same as Eq. (3). These equations need to be solved only for a few $\tau$ steps to populate a narrow band of ghost cells.

\subsection{Vessel Segmentation}

The vessel segmentation was carried by applying the PDE-based local level set method [7] to T1W PCA-MRI velocity data. The reinitialization technique presented in [10] was used, where the following Hamilton-Jacobi type equation;

$$
\frac{\partial d}{\partial \tau}+S\left(d_{0}\right)(|\nabla d|-1)=0,
$$

is solved to steady state, with the initial conditions:

$$
d(\boldsymbol{x}, 0)=d_{0}(\boldsymbol{x})\left\{\begin{aligned}
-2.0 \Delta l & \text { if }\left\|\boldsymbol{u}_{\boldsymbol{m}}\right\| \geq \delta \\
2.0 \Delta l & \text { if }\left\|\boldsymbol{u}_{\boldsymbol{m}}\right\|<\delta
\end{aligned}\right.
$$

where $\boldsymbol{u}_{\boldsymbol{m}}$ is the T1W PCA-MRI velocity vector, $\Delta l$ is the order of $\Delta x$, and $\delta$ is the threshold number. It is sufficient for the level set function (defined as the distance function) to be calculated in the narrow band [7.

It is observed that solving Eq. (4) with initial conditions (5) provides for thinner vessel geometry, not only because PCA-MRI signal tends to be significantly weak in the neighborhood of vessel wall, but also because it is unable to retain the initial position of the interface. We then resolve Eq. (4) with

$$
d(\boldsymbol{x}, 0)=d_{1}(\boldsymbol{x})-\xi \cdot \Delta l
$$


as the initial conditions instead of Eq. (5), where $d_{1}$ is the solution of Eq. (4) with Eq. (5). The choice of $\xi$, typically $0.0<\xi<1.0$, will be discussed in a later section. The distance function $d$ obtained by this procedure is employed as the level set function $\phi$ determining the flow path geometry for the flow field calculation.

\subsection{Boundary Conditions}

Calculations have been performed within a rectangular parallelepiped extracted from the original PCA-MRI data. On the surface of the calculation domain, we need to specify the boundary conditions for both velocity and pressure. The velocity boundary conditions are set equal to the PCA-MRI velocity data. Pressure boundary conditions for the cross section with maximum inlet velocity are set to zero. For the other inlet and all the outlet cross sections, the pressure gradient normal to the calculated boundary surface are set to vanish.

\section{Results}

\subsection{Flow in a Tube: Poiseiulle Flow}

We first calculated the flow field in a straight tube with circular cross section of constant radius. If pressure gradient along the tube is constant and known, the flow is known as a Poiseiulle flow [14. We chose this flow to verify the validity of the zero-velocity fix procedure on the solid wall.

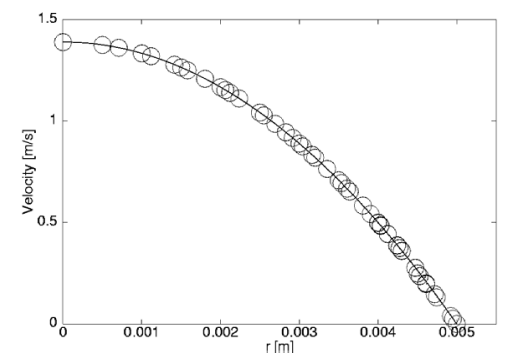

(a) Velocity

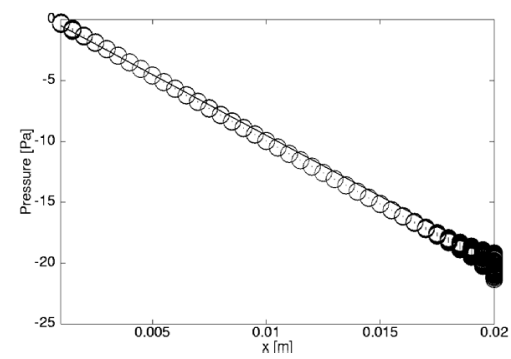

(b) Pressure

Fig. 1. Comparison between theoretical and numerical results for Poiseiulle flow.

Figure 1(a) shows the comparison of the velocity distribution between the theoretical (solid line) and the numerical (open circles) results. Clearly, both curves agree well, confirming that an enforcement of the zero-velocity condition on the tube wall is a reasonably accurate model. These results strongly suggest that the treatment of both isobar and zero-velocity fixes are valid and effective.

Figure 1(b) shows the pressure distribution along the tube. Our numerical scheme employs the zero gradient condition for the outlet pressure, hence the pressure gradient cannot be constant. Discrepancies from theoretical result are 


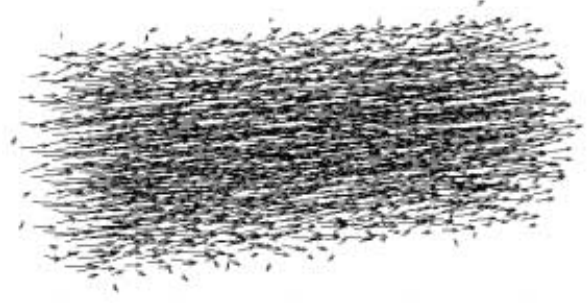

(a) Initial

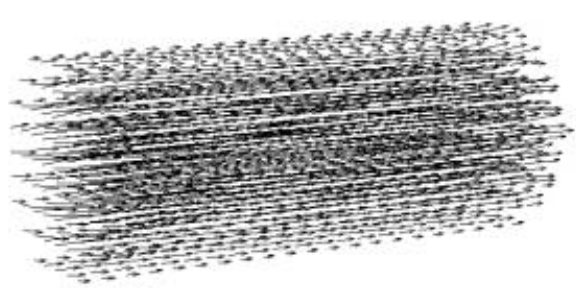

(b) Calculated

Fig. 2. Numerical simulation with contaminated initial condition for Poiseiulle flow (left) and calculation result (right).

therefore inevitable. However, since these discrepancies are sufficiently small, we can verify that our method works well when simulating flow with tubular geometry.

We next evaluated our method with both boundary and initial conditions contaminated with noise. Figure 2(a) shows the synthetic velocity field generated by adding Gaussian white noise to the three components of the velocity field. Figure 2(b) shows the calculated result after 10 time steps. It can clearly be observed that the velocity field is improved with respect to the flow direction, except for the inlet and outlet boundaries where no improvement can be achieved with the current approach. Furthermore, the vessel boundary has not been altered as it would have been with direct smoothing, for example.

\subsection{PCA-MRI Data}

In this section, we present results of applying our method to clinical data. The size of the data set is $256 \times 256 \times 60$, with a field of view (FOV) of $240 \mathrm{~mm}$, slice thickness of $1.5 \mathrm{~mm}$, and velocity encoding of $40 \mathrm{~cm} / \mathrm{s}$. Figure [3] shows the MIP of this image.

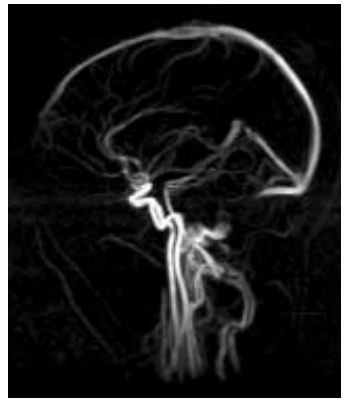

(a) Original MR data

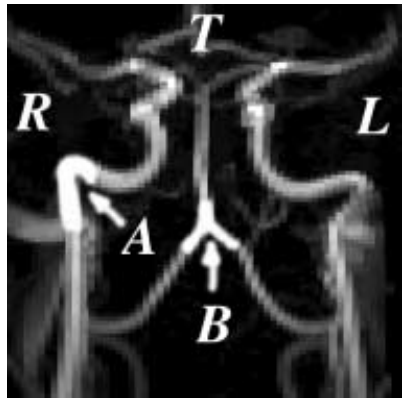

(b) Close up

Fig. 3. Maximum Intensity Projection (MIP) of a PCA-MRI data set. 


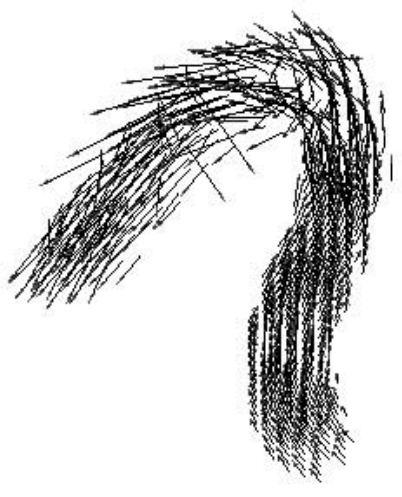

(a) PCA-MRI

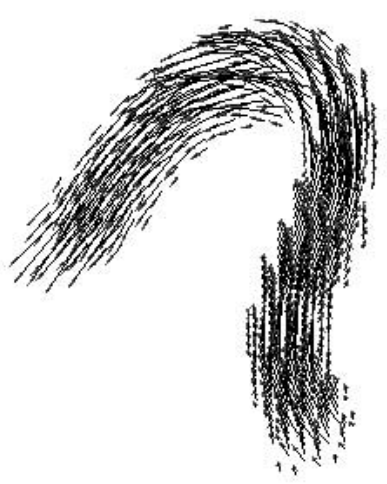

(b) Calculated

Fig. 4. Comparison between PCA-MRI and CFD velocity field for common carotid artery.

After the segmentation procedure described in the previous section, $\phi_{\xi}$ in Eq. (6) is calculated with various $\xi$. This parameter controls the vessel wall location. The velocity field, $\boldsymbol{u}_{\boldsymbol{\xi}}$, for a given $\phi_{\xi}$ and $\boldsymbol{u}_{\boldsymbol{M}}$ can then be calculated. We assume that the most appropriate $\xi$, for a given set of PCA-MRI velocity data, minimizes the discrepancy between the PCA-MRI data and the calculated results. We employ the following expression for this discrepancy, $e$; $e=\sum\left\|\boldsymbol{u}_{\boldsymbol{M}}-\boldsymbol{u}_{\boldsymbol{\xi}}\right\| / \sum\left\|\boldsymbol{u}_{\boldsymbol{M}}\right\|$. The first data set is a section of the common carotid artery (shown as " $A$ " in Fig. [3(b)).

Table 1. Level set correction term for a section of the common carotid artery.

\begin{tabular}{|c||c|c|c|c|c|c|c|c|c|c|}
\hline$\xi$ & 0.0 & 0.2 & 0.3 & 0.4 & 0.5 & 0.6 & 0.7 & 0.8 & 1.0 & 1.5 \\
\hline \hline$e$ & 0.580 & 0.534 & 0.516 & 0.490 & 0.468 & 0.451 & 0.456 & 0.469 & 0.502 & 0.738 \\
\hline
\end{tabular}

The flow field in a bending vessel geometry is calculated. This flow is chosen for the test of both stability and robustness of the method, since the geometry is rather easily segmented. In Table 1 the effect of the level set correction term, $\xi$, on the velocity calculations is shown. We chose $\xi=0.6$, since the discrepancy $e$ is minimum, and calculated results are shown in Fig. 4]

It can be observed that the flow field is significantly improved, especially the direction of velocity vectors which are now naturally aligned along the vessel direction. Notice also that speeds in the original data set, $\left\|\boldsymbol{u}_{\boldsymbol{M}}\right\|$ 's, tend to be greater than those in the calculation, $\left\|\boldsymbol{u}_{\boldsymbol{\xi}}\right\|$ 's, around both elbow and outlet regions. Considering the continuity equation (1), and the velocity distribution around the elbow region shown in Fig. [4(a), it is most possible that the segmentation process provided a thicker vessel diameter around the elbow region. This result also suggests that modification of outlet boundary conditions may provide an improved velocity distribution. 


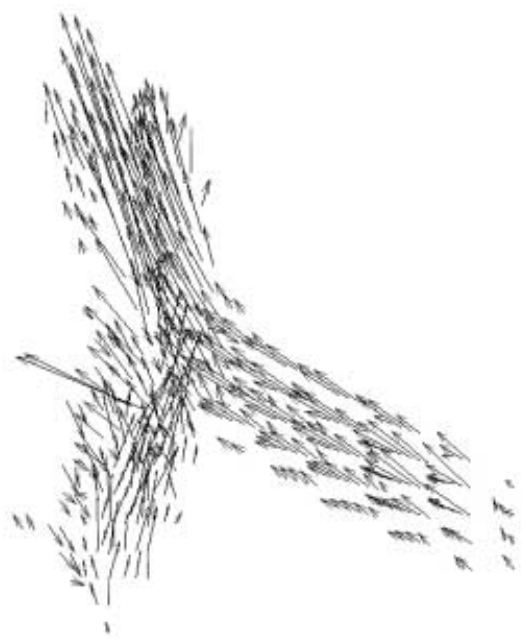

(a) PCA-MRI

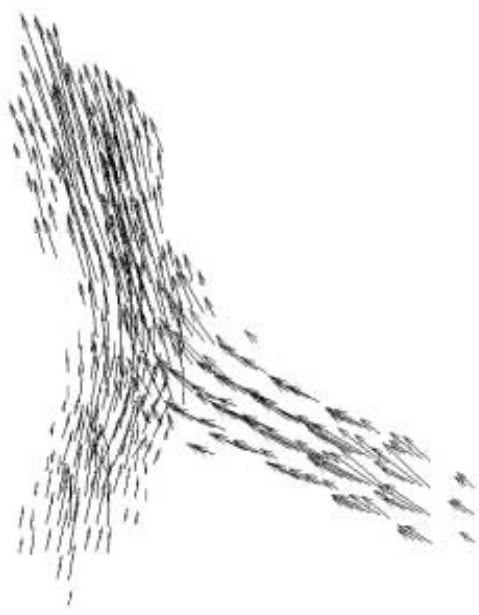

(b) Calculated

Fig. 5. Comparison between PCA-MRI and CFD velocity field for bifurcation of basilar and vertebral arteries.

The second data set is the bifurcation region of the basilar artery and vertebral arteries (shown as " $B$ " in Fig. 3 b)). This is chosen in order to test a more complicated flow than the previous one. The effect of the level set correction term, $\xi$, on the resulting flow is listed in Table 2

Table 2. Level set correction term for bifurcation.

\begin{tabular}{|c||c|c|c|c|c|c|c|}
\hline$\xi$ & 0.0 & 0.1 & 0.2 & 0.3 & 0.4 & 0.5 & 0.6 \\
\hline \hline$e$ & 0.574 & 0.487 & 0.482 & 0.494 & 0.500 & 0.509 & 0.550 \\
\hline
\end{tabular}

It should be emphasized that the optimal value of $\xi$ depends on both vessel geometry and the PCA-MRI signal intensity distribution. The numerical results with $\xi=0.2$ are shown in Fig. 5. Notice that the result is smooth and stable, even with the relatively small amount of data points in our example.

Close to the bifurcation, erroneous velocity can be observed, possibly due to phase wrapping, in Fig. [5(a). These errors are successfully suppressed as shown in Fig. 5(b). Considering the strength of velocity from the right vessel at the bifurcation, it is also possible that the vessel diameter is overestimated, and this information can be employed for the re-segmentation of the vessel.

\section{Conclusion}

A novel correction procedure of PCA-MRI velocity data has been developed, by coupling an incompressible Navier-Stokes equation solver with projection level set GFM, to a PDE-based fast local level set vessel segmentation method. 
Applying this procedure to both synthetic and clinical data, significant improvement of the blood velocity field, such as a smooth velocity distribution aligned along the vessels, and removal of burst or error vectors, could be observed. This procedure also provides possibilities for improved vessel segmentation. The authors are aware of the necessity of more quantitative validation of this procedure, for example, using flow phantom.

\section{Acknowledgments}

This work is supported in part by the International Academic Exchange Foundation of the Faculty of Engineering, Kyushu University (MA), from CIMIT and NIH grant P41-RR13218 (RK, CFW).

\section{References}

1. Leidholdt, E.M.Jr., Bushverg, J.T. Seibert, J.A., Boone, J.M.: The Essential Physics of Medical Imaging, Williams and Wilkins, (1994).

2. Vorp, D.A., Steinman, D.A., Ethier, C.R.: Computational Modeling of Arterial Biomechanics, Comput. Sci. Eng., Vol.3 (5) (2001) 51-64.

3. Milner, J.S., Moore, J.A., Rutt, B.K., Steinman, D.A.: Hemodynamics of Human Carotid Artery Bifurcations: Computational Studies with Models Reconstructed from Magnetic Resonance Imaging of Normal Subjects, J. Vascular Surgery, Vol.28 (1) (1998) 143-156.

4. Lorigo L.M., Faugeras O.D., Grimson W.E.L., Keriven R., Kikinis R., Nabavi A., Westin C.F.: CURVES: Curve Evolution for Vessel Segmentation. Medical Image Analyis. 2001;5:195-206.

5. Long, Q., Xu, X.Y., Ariff, B., Thom, S.A., Hughes, A.D., Stanton, A.V: Reconstruction of Blood Flow Patterns in a Human Carotid Bifurcation: A Combined CFD and MRI Study, J. Mag. Res. Imag., Vol.11 (2000) 299-311.

6. Cebral, J.R., Lohner,R.: From Medical Images to CFD Meshes, Proceedings of the 8th International Meshing Roundtable, (1999) 321-331.

7. Peng, D., Merriman, B., Osher, S., Zhao, H., Kang, M.: A PDE-Based Fast Local Level Set Method, J. Comput. Phys. Vol.155 (1999) 410-438.

8. Fedkiw, R.P., Aslam, T., Merriman, B., Osher, S.: A Non-Oscillatory Eulerian Approach to Interfaces in Multimaterial Flows (The Ghost Fluid Method), J. Comp. Phys., Vol.152. (1999) 457-492.

9. Osher, S., Sethian, J.A.: Fronts Propagating with Curvature Dependent Speed: Algorithms Based on Hamilton-Jacobi Formulations, J. Comput. Phys. Vol.79 (1988) 12-49.

10. Sussman, M., Smereka, P., Osher, S.: A Level Set Approach for Computing Solutions to Incompressible Two-Phase Flos, J. Comput. Phys. Vol. 114 (1994) 146-154.

11. Osher, S., Fedkiw, R.P.: Level Set Methods: An Overview and Some Recent Results, J. Comput. Phys. Vol.169 (2001) 463-502.

12. Sussman, M., Almgren, A. S., Bell, J. B., Colella, P., Howell, L.H., Welcom, M. L.: An Adaptive Level Set Approach for Incompressible Two-Phase Flows, J. Comput. Phys., Vol.148 (1999) 81-124.

13. Fedkiw, R.P.: Coupling an Eulerian Fluid Calculation to a Lagangian Solid Calculation with the Ghost Fluid Method, J. Comp. Phys. (in press) (2002).

14. Batchelor, G.K.: An Introduction to the Fluid Dynamics, Cambridge, (1969). 